\title{
Efficient Analysis of Inductive Multiport Waveguide Circuits using a Surface Integral Equation Formulation
}

\author{
F. D. Quesada Pereira, F. Pérez Soler, B. Gimeno Martínez, V. E. Boria Esbert \\ D. Cañete Rebenaque, J.L. Gómez Tornero and A. Álvarez Melcón \\ Technical University of Cartagena. Campus Muralla del Mar, s/n, E-30202 Cartagena, Spain. E-mail: fernando.quesada@upct.es
}

\begin{abstract}
This paper presents a simple and alternative approach for the analysis of inductive multiport waveguide microwave components. The technique uses a surface integral equation formulation to treat both metallic and dielectric objects inside the component structure. In order to avoid the relative convergence problem of other techniques based on mode matching, a novel port treatment is used. The technique is based on the application of the extinction theorem using the spatial representation of the Green's functions and image theory. Different complex H-plane structures are analyzed, including microwave bandpass filters with elliptic transfer functions and waveguide bends with dielectric posts. Results show the high accuracy and versatility of the new technique derived.
\end{abstract}

\section{INTRODUCTION}

The study of inductive waveguide structures is a subject of intense research, due to the applicability of many waveguide devices in satellite systems and in other microwave high power applications. For the analysis of this type of structures, several approaches were derived in the past, including mode matching techniques [1], finite elements and integral equation formulations. The techniques based on integral equations are usually derived using the spectral representation of the Green's functions inside a parallel plate waveguide [2]. An important limitation of this technique is that all obstacles or coupling windows must be placed inside the parallel plate waveguide considered. Also the input and output ports must be linedup, sharing the based parallel plate waveguide. This limits the possibility to analyze other useful waveguide components such as bends, filters with elliptic responses [3] or T-junctions.

To overcome these difficulties, other authors have used integral equations formulations based on the free space Green's functions. In this case the contour of the base waveguide needs to be discretized, in addition to the inductive posts and windows. The difficulty in this approach is how to model the ports exciting the structure. In [1], [4], [5], this problem was solved by applying mode matching between the waveguide ports and the internal circuit structure. While these approaches are valid, it is well known that care must be taken to avoid relative convergence problems. On the other hand, these approaches open the possibility for the analysis of the more complex microwave devices mentioned above (see Fig. 1).

In this work we present an alternative integral equation technique based on the free space Green's functions and image theory for the port treatment. Both metallic and dielectric objects are treated with an efficient surface formulation. The extinction theorem is further used to separate the internal finite circuit structure from the access ports. An infinite ground

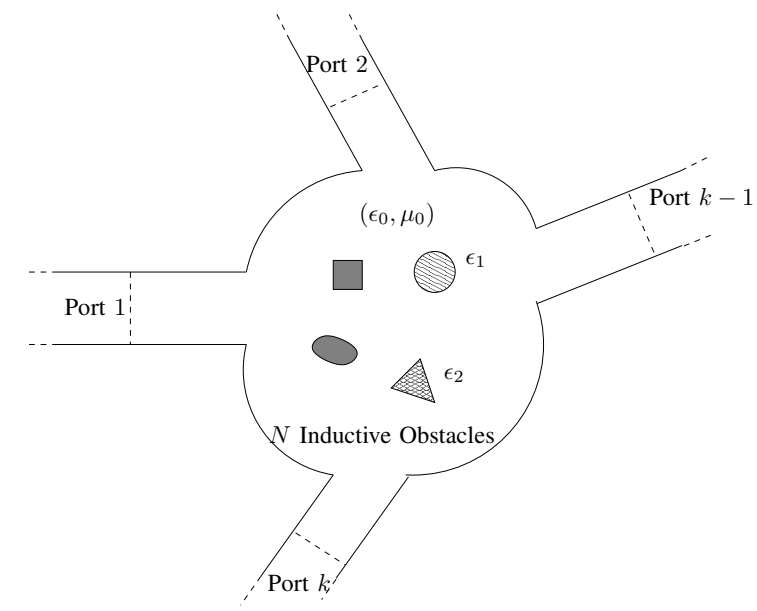

Fig. 1. Typical multiport inductive waveguide problem studied in this paper, containing metallic and dielectric obstacles and $k$ ports.

plane is placed at the exciting port, and it is connected through the external problem to an infinite waveguide section. An additional contribution of the work is the development of an algorithm, based on image theory, so that ports with arbitrary orientations can be treated. A third contribution of the work is that the Green's functions inside the infinite waveguide port are formulated directly in the spatial domain. This is done, for the first time, by combining the spectral and spatial representations of the Parallel Plate Waveguide (PPW) Green's functions. Also, the Kummer [6] and Ewald [7] acceleration techniques are combined when appropriate, for the first time, for maximum efficiency. The new algorithm derived has proved useful in the analysis of bends and Tjunctions.

\section{TheORETICAL OUtLine}

The analysis of a general inductive waveguide multiport structure shown in Fig. 1 is analyzed using an integral equation formulation. The technique is based on the evaluation of the short-circuited admittance parameters $(Y)$ of the multiport structure. This is done by alternatively considering an incoming excitation wave on each port $(j)$, and shortcircuiting all others $(i)$. For each case the electric and magnetic fields on the ports have to be evaluated. Then, the final $Y$ parameters are computed through integration of the fields with 
the corresponding modal functions:

$$
Y_{m, n}^{(i, j)}=\frac{\iint_{S_{i}} \bar{H}_{t}^{(i)} \cdot \bar{h}_{m}^{(i)} d S}{\iint_{S_{j}} \bar{E}_{t}^{(j)} \cdot \bar{e}_{n}^{(j)} d S}
$$

For the computation of the fields, a surface integral equation is formulated. The integral equation is applied to arbitrarily shaped inductive metallic and dielectric objects, by defining appropriate equivalent electric and magnetic currents on the object countours. For the discretization of the integral equation, triangular roof-top type functions defined on linear segments are used. This leads to high accuracy for the representation of arbitrarily complex shaped metallic and dielectric objects. The exciting input port is futher isolated from this analysis by using the extinction theorem (see Fig. 2). It can be seen that an infinite ground plane is placed at the reference plane to short-circuit any port equivalent electric currents (Fig. 2).

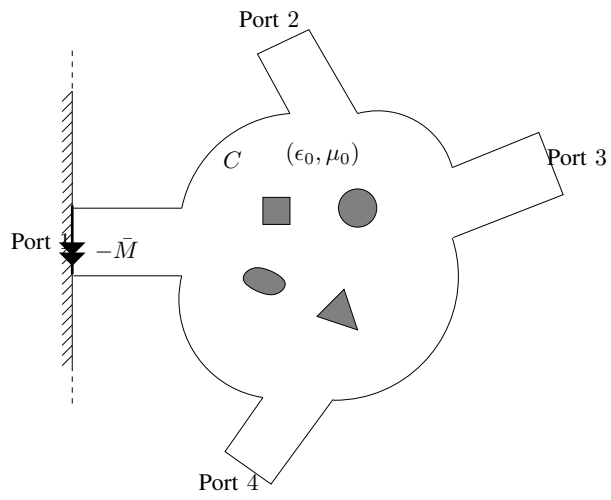

Fig. 2. Internal circuit problem resulting from the application of the extinction theorem.

For the solution of this problem the Green's functions to be used are those in the presence of an infinite ground plane. The Green's functions can therefore be easily formulated using image theory (see the appendix). In this work, a procedure has been derived for the calculation of the Green's functions in the presence of an arbitrarily oriented ground plane (see Fig. 3).

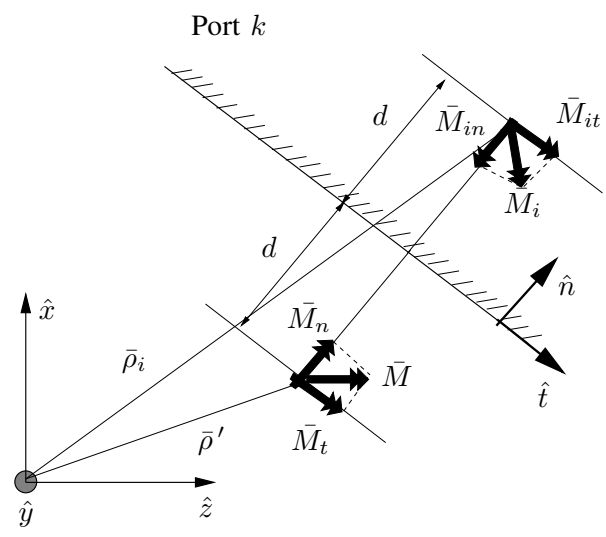

Fig. 3. Source and image magnetic dipoles for an arbitrarily oriented ground plane.

The formulation derived for arbitrary orientations has proved useful in the analysis of inductive bends and Y-junctions, when one port can be placed at reference axis with different slopes.

On the other hand, the external port problem assumes an incoming wave in a semi-infinite waveguide as shown in

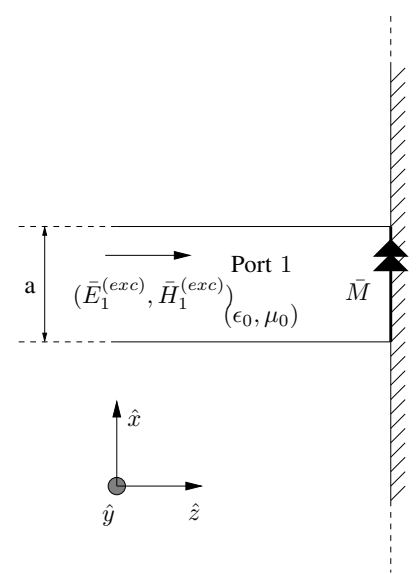

Fig. 4. External port problem resulting from the application of the extinction theorem.

Fig. 4. The coupling to the internal problem is accomplished by placing equivalent magnetic currents at the reference plane. In this case, a magnetic current density equal to $2 \bar{M}$ is assumed in order to eliminate the ground plane. The resulting problem is, therefore, reduced to the evaluation of the Green's functions for magnetic currents inside an infinite parallel plate waveguide (PPW). The main difficulty for the evaluation of these Green's functions is that they are formulated either as modal series or as spatial images series, both exhibiting very slow convergence behaviors. Some approaches in the past have used spectral representations of the series with a particular choice of the basis functions to accelerate convergence [8]. Instead, in this work we have preferred to obtain a representation of the Green's functions directly in the spatial domain. We have done this by combining for the first time the spectral and spatial formulations of the PPW Green's functions, together with a combination of series acceleration techniques. In order to increase the efficiency of the algorithm, we have combined when appropriate, for the first time, the Kummer and the Ewald techniques to accelerate the convergence rate of the Green's functions series. The advantage of having the Green's functions in the spatial domain, is that the same discretization scheme used for the internal circuit problem can also be used in the characterization of the ports. This strategy has shown to increase the flexibility of the technique. In addition, the relative convergence problem of other mode matching techniques [1], [4] is avoided with the proposed formulation.

\section{RESULTS}

The software developed based on the technique presented finds applications in the analysis of many useful microwave circuits. The first interesting structures that we investigate are the all inductive elliptic filters presented in [3]. Since all the cavities of the resonators have different widths, these structures can not be analyzed using a simple parallel plate Green's functions approach [2]. However, we can easily use the technique presented in this contribution.

As a first result, we present a filter with one dual mode cavity. The internal circuit is discretized with 175 segments, and then the coupling to the waveguide ports is modelled using 15 basis functions. Fig. 5 shows the results obtained for the scattering parameters of the filter. Measured results presented 


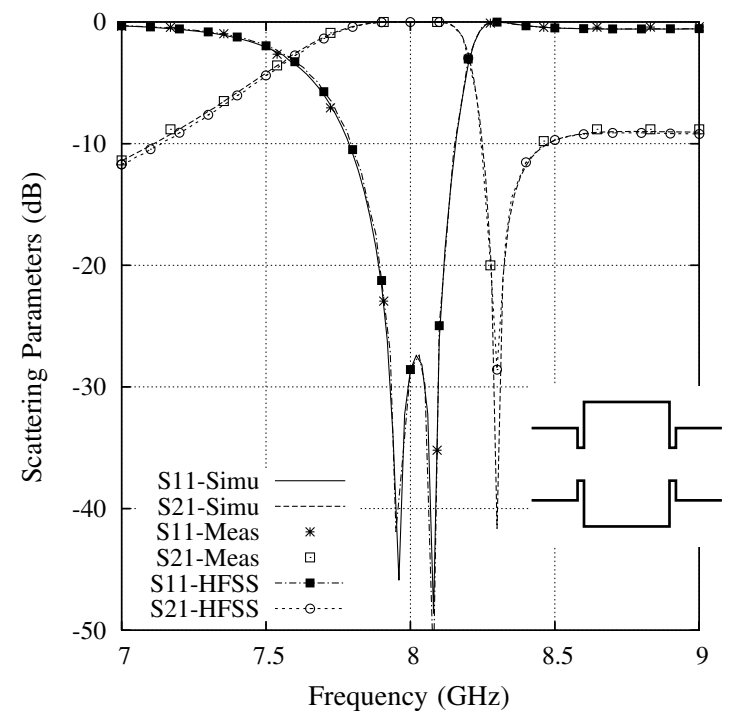

Fig. 5. Scattering parameters of a filter with one dual mode cavity. Dimensions are shown in [3]-Fig.4.

in [3], and simulations obtained with $\mathrm{HFSS}^{1}$ are also given, showing good agreement with our new simulation tool. For this structure the software takes 5.8 seconds per frequency point on a PC computer with $1.6 \mathrm{GHz}$ clock.

A more complex filter with two dual mode cavities exhibiting elliptic response was designed in [3]. Fig. 6 shows the measured and simulated results calculated with the new approach. Reference results obtained with HFSS are also included. We can see that the agreement obtained with the new

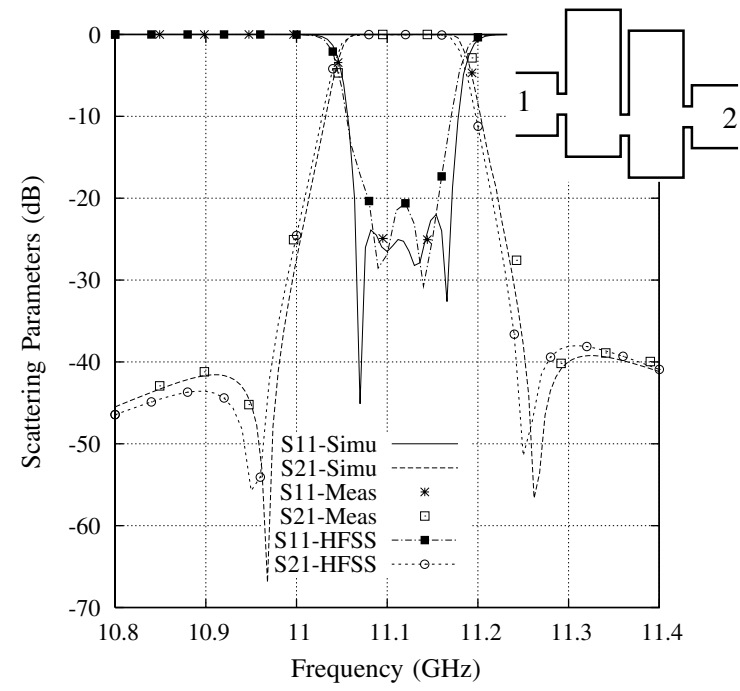

Fig. 6. Scattering parameters of a filter with two dual mode cavities. Dimensions are shown in [3]-Fig.6.

technique is good, for both measurements and HFSS results. A total of 241 basis functions were used for the discretization of the internal circuit topology, and 15 basis functions for the ports. The software takes 9.2 seconds per frequency point on a PC computer with $1.6 \mathrm{GHz}$ clock.

The multiport nature of the tool developed can be best discussed with the analysis of a T-junction, where three ports must be treated at the same time. In this case we have selected a T-junction compensated by a cylindrical metallic

${ }^{1}$ HFSS v.10.0, Ansoft Corporation, Pittsburgh, PA, 2005. post, presented in [1]. Furthermore, we have evaluated the compensation capabilibities of a dielectric post placed in addition to the original metallic post. Fig. 7 presents the results obtained for the reflection coefficient at the common port, when the additional dielectric post is used for compensation. Also the original results using the single metallic post are presented. Reference results obtained with HFSS and measurements are again included for comparison. It can be seen that

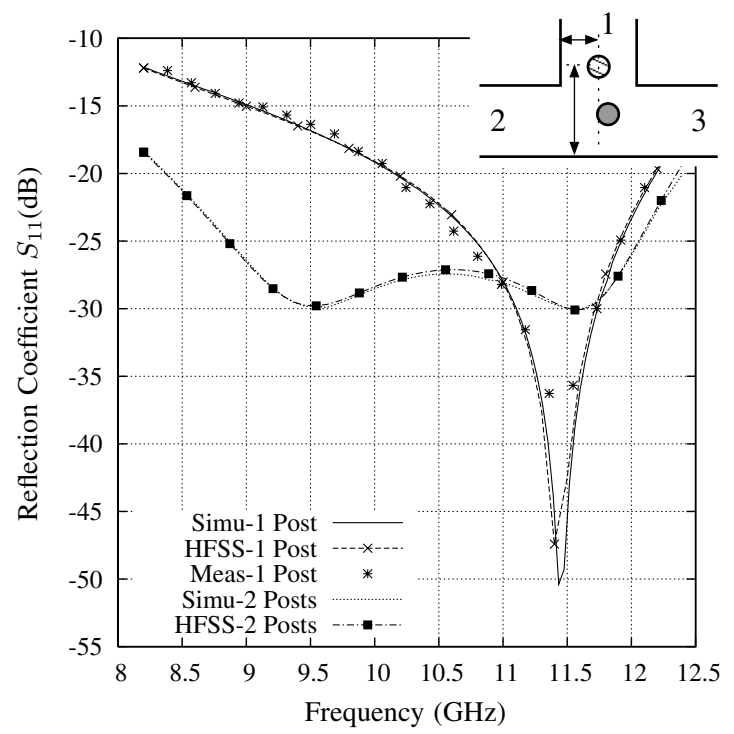

Fig. 7. Reflection coefficient of the common port for the T-junction presented in [1]-Fig.2. The new structure with two posts has the dielectric: $\epsilon_{r}=5$, radius $=0.75 \mathrm{~mm}$, position $(11.43 \times 28.86) \mathrm{mm}$; metallic post has: radius $=1.10 \mathrm{~mm}$, position $(11.53 \times 8.86) \mathrm{mm}$.

the coupling to the additional dielectric post can considerably widen the matching bandwidth of the device, as compared to the original single metallic post structure. For this analysis, 169 basis functions are used for the circuit and 12 for the ports. The software takes 9 seconds per frequency point on the same computer as before.

An additional example is presented for a mitered bend with a square dielectric post first introduced in [9]. Fig. 8 shows the transmitted power through the bend for the four cases considered in [9] (with different dielectric sizes and materials). Results reported in [9] and HFSS data are also included for

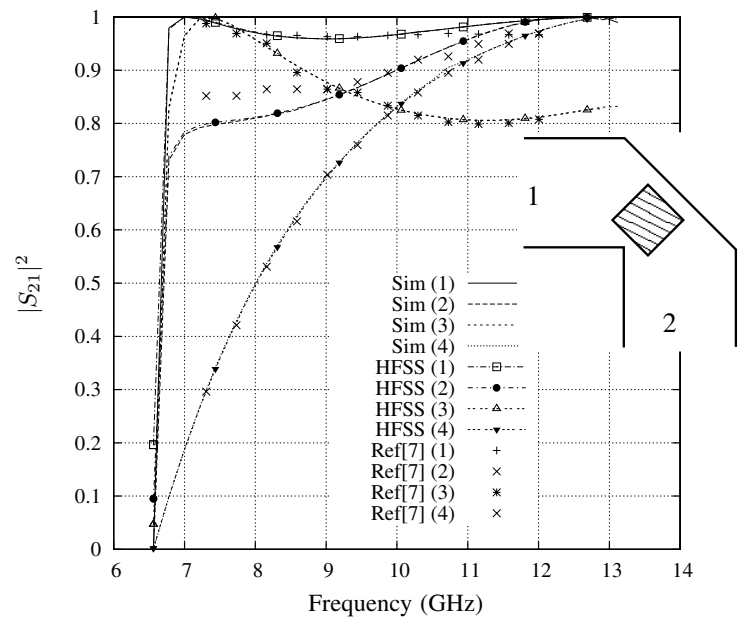

Fig. 8. Normalized transmitted power for the four dielectric configurations of the mittered bend presented in [9]-Fig4 (bend width=22.86mm). 
comparison. Again good agreement is obtained using the new technique proposed in this paper. For the analysis of this structure a total of 158 basis functions were used for the circuit. The software takes 4.7 seconds per frequency point on the same computer.

In all above examples, the ports are all oriented either horizontally or vertically. In order to validate the port treatment for different orientations, we have further analyzed an inductive bend with different bend-angles, as presented in [1]. Fig. 9 shows the reflection coefficient when the angle of the bend is varied from $90^{\circ}$ to $150^{\circ}$. Results reported in [1] and results obtained with HFSS are given for comparison. Again good

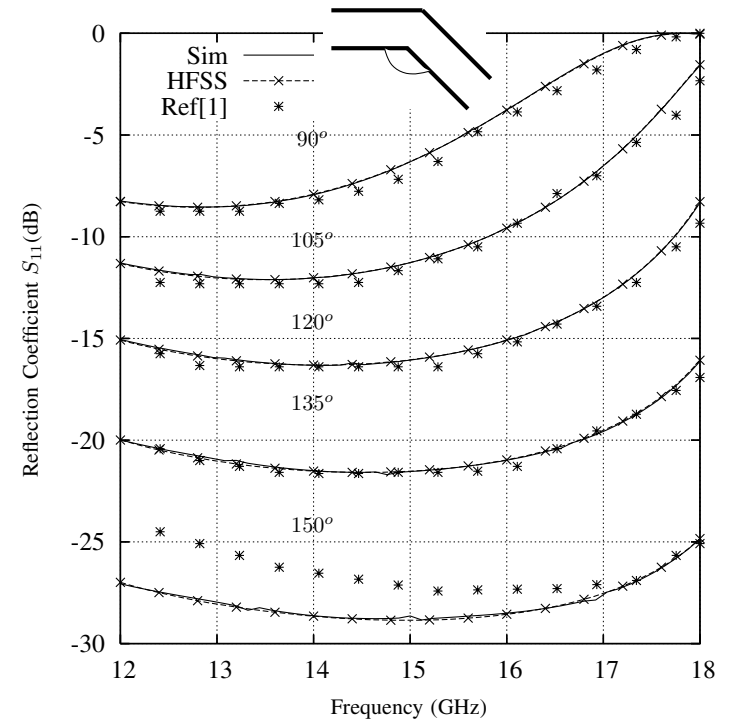

Fig. 9. Reflection coefficient for bends with varying angle from $90^{\circ}$ to $150^{\circ}$ Dimensions are shown in [1]-Fig.3a.

agreement is obtained for angle bends smaller than $135^{\circ}$. When the angle is $150^{\circ}$, our results agree better with those obtained using the Finite Elements technique (HFSS). For the analysis of this structure a total of 83 basis functions were used for the circuit and 15 for the ports. The software takes 1.9 seconds per frequency point on the same computer.

\section{CONCLUSIONS}

In this paper an alternative surface integral equation formulation is proposed for the analysis of complex inductive waveguide microwave devices, composed of both metallic and dielectric objects. The technique uses the extinction theorem to separate the internal circuit problem from the input/output ports. A formulation has been presented to express the Green's functions of the internal circuit problem with respect to an arbitrarily oriented ground plane. For the external port problem the infinite parallel plate waveguide Green's functions of magnetic type are used. Unlike traditional approaches, the Green's functions are obtained in the space domain by combining Kummer and Ewald series acceleration techniques. Results are presented for several practical microwave devices demonstrating the accuracy and efficiency of the new technique.

\section{APPENDIX}

Based on the ideas presented in Sección II, the final relevant Green's functions components needed in the formulation can be written, using the auxiliary vectors shown in Fig. 3, as:

$$
\begin{aligned}
& G_{A}^{y y}\left(\vec{\rho}, \vec{\rho}^{\prime}\right)=\frac{\mu_{0}}{4 j}\left[H_{0}^{(2)}\left(k_{0}\left|\vec{\rho}-\vec{\rho}^{\prime}\right|\right)-H_{0}^{(2)}\left(k_{0}\left|\vec{\rho}-\vec{\rho}_{i}\right|\right)\right] \\
& G_{W}\left(\vec{\rho}, \vec{\rho}^{\prime}\right)=\frac{1}{4 j \mu_{0}}\left[H_{0}^{(2)}\left(k_{0}\left|\vec{\rho}-\vec{\rho}^{\prime}\right|\right)+H_{0}^{(2)}\left(k_{0}\left|\vec{\rho}-\vec{\rho}_{i}\right|\right)\right] \\
& G_{F}^{x x}\left(\vec{\rho}, \vec{\rho}^{\prime}\right)=\frac{\epsilon_{0}}{4 j}\left\{H_{0}^{(2)}\left(k_{0}\left|\vec{\rho}-\vec{\rho}^{\prime}\right|\right)+\right. \\
& \left.\left[H_{0}^{(2)}\left(k_{0}\left|\vec{\rho}-\vec{\rho}_{i}\right|\right)(\hat{t} \cdot \hat{x}) \hat{t}-H_{0}^{(2)}\left(k_{0}\left|\vec{\rho}-\vec{\rho}_{i}\right|\right)(\hat{n} \cdot \hat{x}) \hat{n}\right] \cdot \hat{x}\right\}
\end{aligned}
$$

$$
\begin{aligned}
G_{F}^{z x}\left(\vec{\rho}, \vec{\rho}^{\prime}\right)=\frac{\epsilon_{0}}{4 j}\{[ & H_{0}^{(2)}\left(k_{0}\left|\vec{\rho}-\vec{\rho}_{i}\right|\right)(\hat{t} \cdot \hat{x}) \hat{t}- \\
& \left.\left.H_{0}^{(2)}\left(k_{0}\left|\vec{\rho}-\vec{\rho}_{i}\right|\right)(\hat{n} \cdot \hat{x}) \hat{n}\right] \cdot \hat{z}\right\}
\end{aligned}
$$

$$
\begin{aligned}
& G_{F}^{x z}\left(\vec{\rho}, \vec{\rho}^{\prime}\right)=G_{F}^{z x}\left(\vec{\rho}, \vec{\rho}^{\prime}\right) \\
& G_{F}^{z z}\left(\vec{\rho}, \vec{\rho}^{\prime}\right)=\frac{\epsilon_{0}}{4 j}\left\{H_{0}^{(2)}\left(k_{0}\left|\vec{\rho}-\vec{\rho}^{\prime}\right|\right)+\right. \\
& \left.\left[H_{0}^{(2)}\left(k_{0}\left|\vec{\rho}-\vec{\rho}_{i}\right|\right)(\hat{t} \cdot \hat{z}) \hat{t}-H_{0}^{(2)}\left(k_{0}\left|\vec{\rho}-\vec{\rho}_{i}\right|\right)(\hat{n} \cdot \hat{z}) \hat{n}\right] \cdot \hat{z}\right\}
\end{aligned}
$$

\section{REFERENCES}

[1] J. M. Reiter and F. Arndt, "Rigorous analysis of arbitrarily shaped H- and E-plane discontinuities in rectangular waveguides by a full wave boundary contour mode matching method," IEEE Transactions on Microwave Theory and Techniques, vol. 43, pp. 796-801, April 1995.

[2] H. Auda and R. F. Harrington, "Inductive posts and diaphragms of arbitrary shape and number in a rectangular waveguide," IEEE Transactions on Microwave Theory and Techniques, vol. 32, pp. 606-613, June 1984

[3] M. Guglielmi, P. Jarry, E. Kerherve, O. Roquebrun, and D. Schmitt, "A new family of all-inductive dual-mode filters," IEEE Transactions on Microwave Theory and Techniques, vol. 49, pp. 1764-1769, October 2001.

[4] H. Esteban, S. Cogollos, V. Boria, A. A. San Blas, and M. Ferrando, "A new hybrid mode-matching/numerical method for the analysis of arbitrarily shaped inductive obstacles and discontinuities in rectangular waveguides," IEEE Transactions on Microwave Theory and Techniques, vol. 50, pp. 1219-1224, April 2002.

[5] V. Catina, F. Arndt, and J. Brandt, "Hybrid surface integral equation/mode-matching method for the analysis of dielectric loaded waveguide filters of arbitrary shape," IEEE Transactions on Microwave Theory and Techniques, vol. 53, pp. 3562-3567, November 2005.

[6] Y. Leviatan, P. G. Li, A. T. Adams, and J. Perini, "Single post inductive obstacle in rectangular waveguide," IEEE Transactions on Microwave Theory and Techniques, vol. 31, pp. 806-812, October 1983.

[7] F. Capolino, D. R. Wilton, and W. A. Johnson, "Efficient computation of the 2-D Green's function for 1-D periodic structures using the ewald method," IEEE Transactions on Antennas and Propagation, vol. 53, pp. 2977-2984, September 2005.

[8] P. G. Li, A. T. Adams, Y. Leviatan, and J. Perini, "Multiple post inductive obstacles in rectangular waveguide," IEEE Transactions on Microwave Theory and Techniques, vol. 32, pp. 365-373, April 1984

[9] K. L. Wu, G. Y. Delisle, D. G. Fang, and M. Lecours, "Waveguide discontinuity analysis with a coupled finite boundary element method," IEEE Transactions on Microwave Theory and Techniques, vol. 37, pp. 993-998, June 1989. 